\title{
Infiltration of a photonic crystal fiber with cholesteric liquid crystal and blue phase
}

\author{
D. Poudereux ${ }^{* a}$, K. Orzechowski ${ }^{\mathrm{b}}$, O. Chojnowska ${ }^{\mathrm{c}}$, M. Tefelska ${ }^{\mathrm{b}}$, T. R. Woliński ${ }^{\mathrm{b}}$, J. M. Otón ${ }^{\mathrm{a}}$ \\ ${ }^{a}$ CEMDATIC, ETSI Telecomunicación, Universidad Politécnica de Madrid, Av. Complutense nº 30, \\ 28040, Madrid, Spain \\ ${ }^{b}$ Faculty of Physics, Warsaw University of Technology, Koszykowa 75, 00-662 Warsaw, Poland \\ ${ }^{c}$ Institute of Chemistry, Military University of Technology, Kaliskiego 2, 00-908 Warsaw, Poland
}

\begin{abstract}
Photonic crystal fibers (PCF) have been selectively filled with a cholesteric liquid crystal (ChLC) with special interest in the blue phase (BP) of the liquid crystal. It has been observed thermal tuning of the guided light in the visible region. A dramatically enhance appears when the phase of the liquid crystal changes from cholesteric to blue phase I (BPI). When a thermal range of the blue phase I is achieved, no changes are observed while increasing temperature from BPI through BPII and to the isotropic phase.
\end{abstract}

Keywords: Photonic crystal fiber, liquid crystal, cholesteric liquid crystal, blue phase, selectively infiltration.

\section{INTRODUCTION}

Photonic crystal fibers (PCFs) are fibers with periodically distributed air holes parallel to the fiber axis. These special fibers are very versatile and can be designed for different purposes changing the geometry, shape and periodicity of the holes [1],[2]. Moreover the passive properties of the PCFs can be enhanced by filling these holes with materials having active optic properties like liquid crystals (LCs) [3]-[5] forming the named photonic liquid crystal fibers (PLCF). PCFs can guide the propagating light by total internal reflection (TIR) as in standard optical fibers or by photonic band-gap (PBG) where only certain ranges of wavelengths are guided and the others are not. In the second case, the refractive index of the core must be lower than the refractive index of the cladding. Usually both LC refractive indices, ordinary and extraordinary, are higher than the refractive index of the fiber cladding. In this case, guiding is only achieved by photonic band-gap (PBG). If the fiber holes are filled with a LC having one of their refractive indices lower than the effective refractive index of the fiber cladding (usually silica), it is possible to switch from one light guiding mechanism to the other [6]. Cholesteric (or chiral nematic) liquid crystal resembles nematic liquid crystal in all physical properties except that the molecules tend to arrange themselves in a helical structure with the helical axis perpendicular to the director. The length over which the LC director rotates $2 \pi$ is defined as helical pitch, which indicates the twist extent of LC molecules. It was proposed by Tefelska et al. an optical filtering by temperature-induced tuning of the photonic band gaps in a PCF infiltrated with cholesteric LC [7].

Cholesteric liquid crystals present a blue phase (BP) in a narrow range of temperature. This chiral mesophase is formed by a 3D fluid unity cell with cubic defects and has the property to be macroscopically isotropic but exhibiting Bragg reflections. There are in fact three blue phases. Blue phase I has a body centered unity cell formed with double-twisted cylinders, blue phase II is simple cubic and blue phase III is a foggy phase very difficult to distinguish with the isotropic phase. It has been proved that blue phases have a hysteresis in the transition from cholesteric phase to blue phase [8],[9]. Recently it has been developed a polarization-independent bistable light valve in a photonic crystal fiber [10] using this hysteresis effect. It has been reported that blue phase as fiber waveguiding cores are optically isotropic, polarization insensitive and relatively low-loss [11]. Khoo et al. show that because of the tightly-wound director axis arrangement in blue phase, the scattering loss associated with director axis fluctuations is largely reduced and allows good transmission through long (several mm's) interaction lengths. Blue phase LC is a promising material to fill photonic crystal fiber because the molecules in this phase are self-aligned, without need of an alignment layer.

*dpoudere@gmail.com

Photonics Applications in Astronomy, Communications, Industry, and High-Energy Physics

Experiments 2014, edited by Ryszard S. Romaniuk, Proc. of SPIE Vol. 9290, 92900A

(C) 2014 SPIE · CCC code: $0277-786$ X/14/\$18 $\cdot$ doi: $10.1117 / 12.2074525$

Proc. of SPIE Vol. $929092900 A-1$ 
The alignment of the molecules inside cylindrical microcavities is not a trivial matter. It could be three different configurations [12] depending in the surface anchoring force (Fig. 1). If the anchoring force is strong the helix will disappear because the molecules are aligned parallel to the surface with the director parallel to the fiber axis. If the anchoring is weak the molecules will align perpendicular (homeotropic) to the surface forming the helix parallel to the fiber axis. In the last configuration the molecules are planar aligned but whit the helix perpendicular to the fiber axis. This alignment is similar to the double twisted cylinders which forms the unity cell in the blue phase.

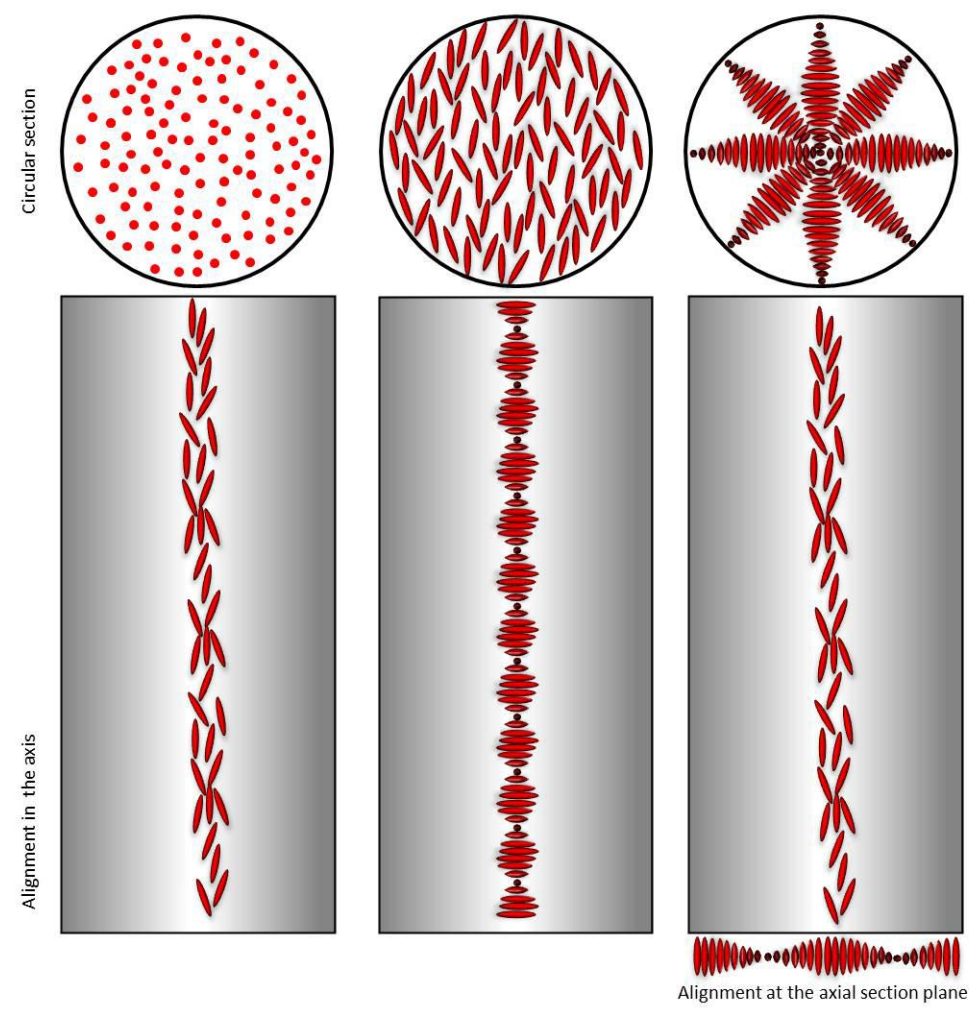

Figure 1: Possible configuration of the cholesteric molecules alignment inside of a cylindrical microcavity. Left: strong anchoring induces planar alignment with unwound helix. Middle: weak anchoring with a homeotropic alignment and with helix along the fiber. Right: planar alignment with the helix perpendicular to the fiber.

Several ways has been developed to selectively fill a PCF. It could be made by blocking the desired holes of the fiber one by one with optical adhesive [13],[14], by capillary filling with optical adhesive [15] and using a standard fusion splicer. For the last two cases it is necessary an enough difference in diameter size of the holes.

In this work a commercial PCF developed by Thorlabs (named PM-1550-01) has been selectively filled with cholesteric liquid crystal (ChLC) and blue phase. Increasing the temperature the phase of the liquid crystal is changed from cholesteric to blue phase, showing a dramatically increasing of the intensity power in the transition point. Once the blue phase I is achieved no change it is seen when the phase change from blue phase I to blue phase II and finally isotropic phase.

\section{MATERIALS AND METHODS}

The selected photonic crystal fiber, PM-1550-01 (Fig. 2) manufactured by Thorlabs, is a polarization maintaining PCF made in pure silica. It has two big holes of $4.5 \mu \mathrm{m}$ in the same diagonal close to the solid core surrounded by 5 rings of small holes of $2.4 \mu \mathrm{m}$ forming the cladding. 


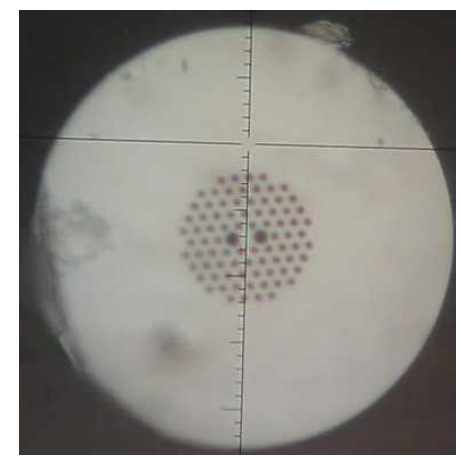

Figure 2: Polarization maintaining fiber PM-1550-01 by Thorlabs

The difference on the diameter size of the holes and the geometry of this PCF made possible the selective cladding collapsing with a standard fusion splicer remaining the bigger holes open ready to be infiltrated with the desired material, in this case a cholesteric liquid crystal. The cholesteric liquid crystal, named BP 2029 A, was manufactured in the Military University of TechnologyWarsaw with special emphasis on its blue phase. The specifications are detailed in table 1.

Table 1. Phase transition temperatures of the BP 2029A LC; $\mathrm{N}^{*}$ stands for the cholesteric phase and BPI and BPII are blue phase I and II

\begin{tabular}{|l|l|}
\hline \multicolumn{2}{|c|}{ BP 2029A } \\
\hline Heating & $\mathrm{N}^{*}$ 38.9 BPI 39.9 BPII 43.0 Iso \\
\hline Cooling & Iso 24.2 BPII 39.8 BPI 28.5 N* \\
\hline
\end{tabular}

To selectively collapse the small holes of the cladding a standard fusion splicer Ericsson FSU 995 PM was used. Once the small holes are collapsed remaining only the two big holes close to the core the fiber is ready to be filled with the liquid crystal by capillary. The collapsed side of the fiber is submerged into the fluid and then a red laser is launched in the fiber to control the length of the filled. The spot formed by the scattered light is visible with the naked eye and moves slowly across the fiber leaving a track of scattered light in the filled fiber. To fill a region of $1.5 \mathrm{~cm}$ is necessary around 8 minutes to fill the fiber with the liquid crystal in isotropic phase, at $50^{\circ}$ (Fig. 3). Once the fiber is filled it is spliced after the collapsed region to do the measurements.

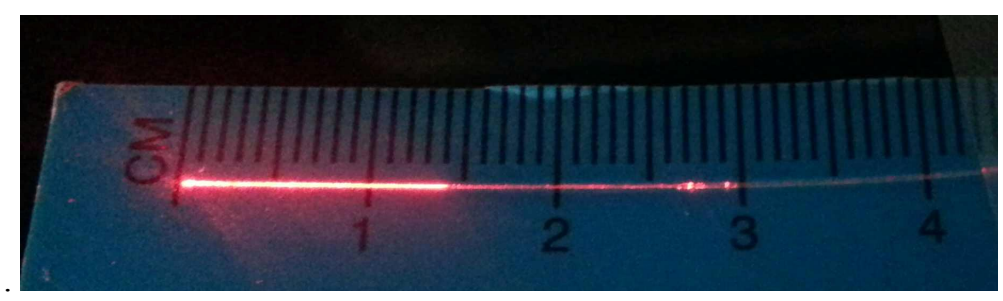

Figure 3: Scattered light of the region infiltrated with liquid crystal

Before cut the fiber is possible to observe the collapsed region under the microscope between crossed polarizers to see the alignment of the LC molecules inside the holes. As only the two big holes are filled and the cladding holes are collapsed there are not scattering produced by the cladding (Fig. 4). 

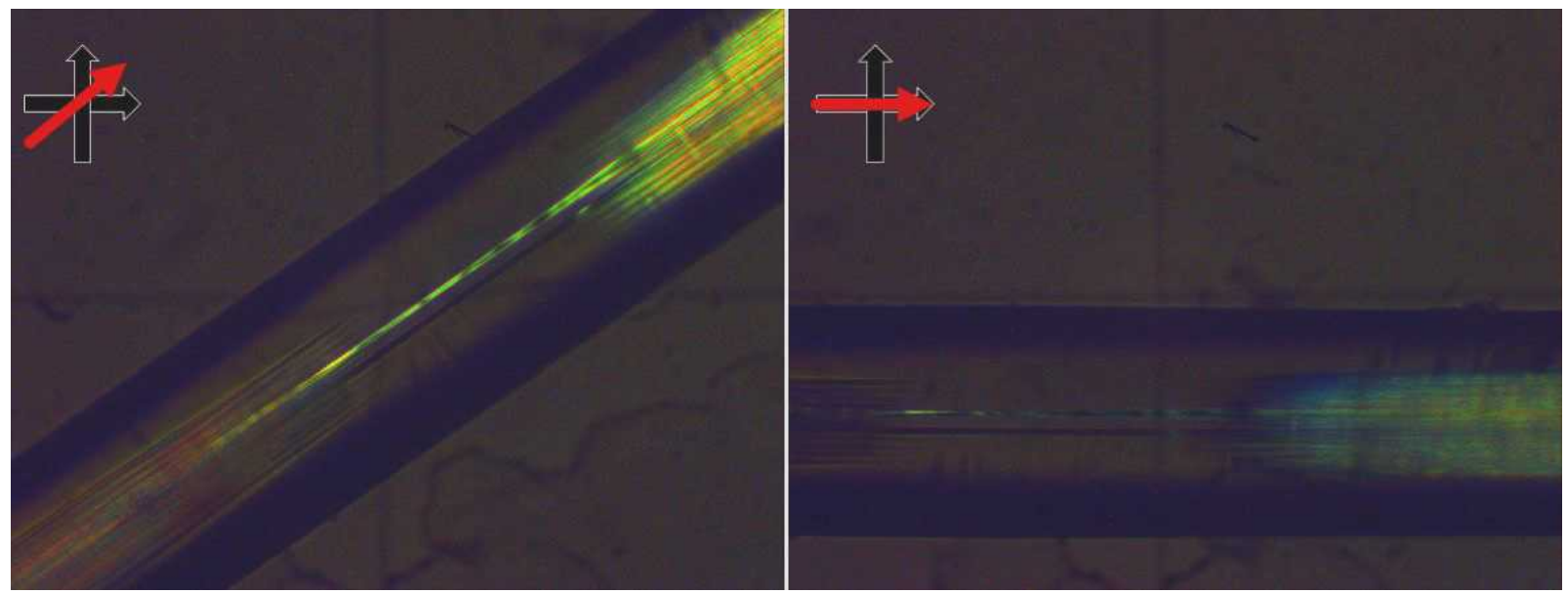

Figure 4: Selectively filled PM1550-01 with BP 2020A with the cladding collapsed under a microscope between crossed polarizers. Left: at $45^{\circ}$ to both polarizers, Right: parallel to one of the polarizers

In Fig. 4 it is seen a partially filled PM-1550-01 by its right side. In the center region of the fiber all smaller holes of the cladding are collapsed remaining only the two bigger. Only one of the two bigger holes is filled due to see the difference between them. In the right side of the fiber all holes are filled but after the collapsed region, on the left side, only one big hole is filled. A softy scattering of the light produced by the filled big hole it is seen on the left side.

When the fiber is at $45^{\circ}$ to both polarizers it is seen a maximum in the transmitted light. This is a sign of the aligning of the molecules, but it is no possible to see if the alignment is perpendicular or parallel to the inner surfaces of the holes. These pictures were taken at room temperature, for this reason it is reasonable the assumption that the liquid crystal is in cholesteric phase instead of blue phase.

Rotating the sample $45^{\circ}$, parallel to one on polarizer, the transmitted light is minimized. In this case some regions are a little brighter than other suggesting that the alignment of the molecules is not totally homogenous. The intensity should be totally dark without brighter zones if the alignment of the molecules where absolutely parallel to the axis of the fiber.

\section{RESULTS}

To observe thermal tunability of the guided light, a white light source in the visible region was launched into the PCF and the output side was observed with a microscope and a camera. The filled region, around $10 \mathrm{~mm}$ was heated from room temperature at $23^{\circ} \mathrm{C}$ to $50^{\circ} \mathrm{C}$ with a Peltier module. The initial phase of the liquid crystal is cholesteric and the pitch of this phase change with the temperature resulting in a change in the color of the output light. When the fiber PM1550-01 is empty the fundamental mode has a shape of an elongated spot in the core[16]. As the big holes are filled with a material with higher refractive index than silica the fundamental mode splits in two spots close to the core (Fig. 4).

By increasing temperature we observe a dramatical change of the color and intensity near the blue phase I transition temperature. After this point the transmitted light intensity reaches its maximum and no more changes neither in color nor in intensity are observed. As this behavior is according to an isotropic phase the assumption that this is blue phase it is presumably correct. 

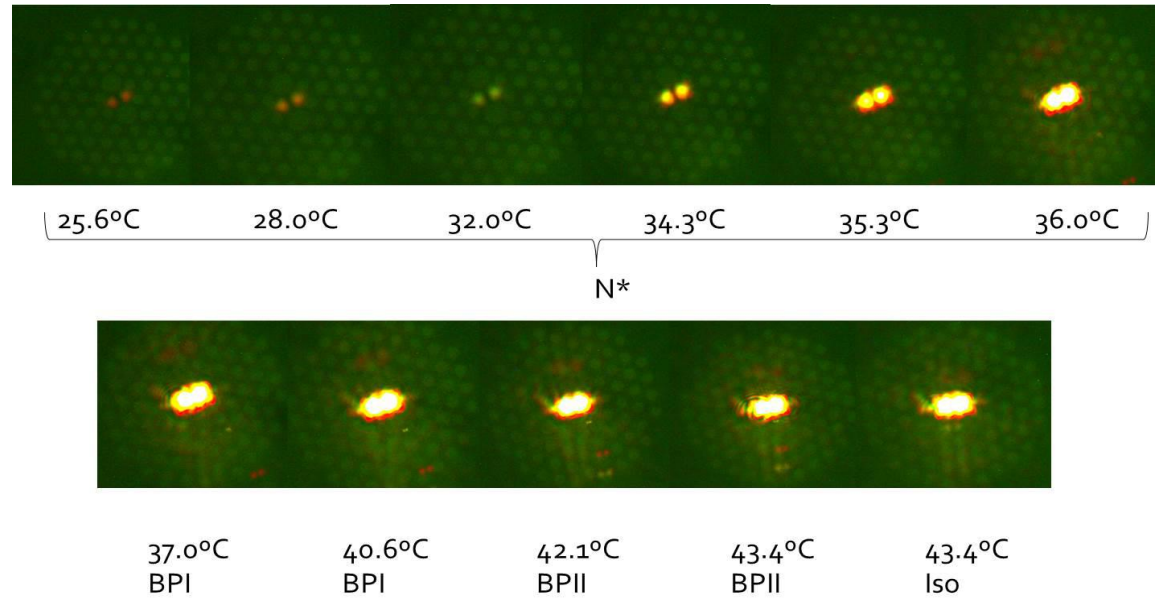

Figure 5: Thermal tunability of the guided light of a white source in the PM-1550 PCF selectively filled with cholesteric LC

We have also performed measurements by using a tunable laser in the optical communications windows between 1500 $\mathrm{nm}$ and $1600 \mathrm{~nm}$. The temperature range was the same, between room temperature and around $60^{\circ} \mathrm{C}$. It is showed in Fig. 6 that intensity increases with temperature.

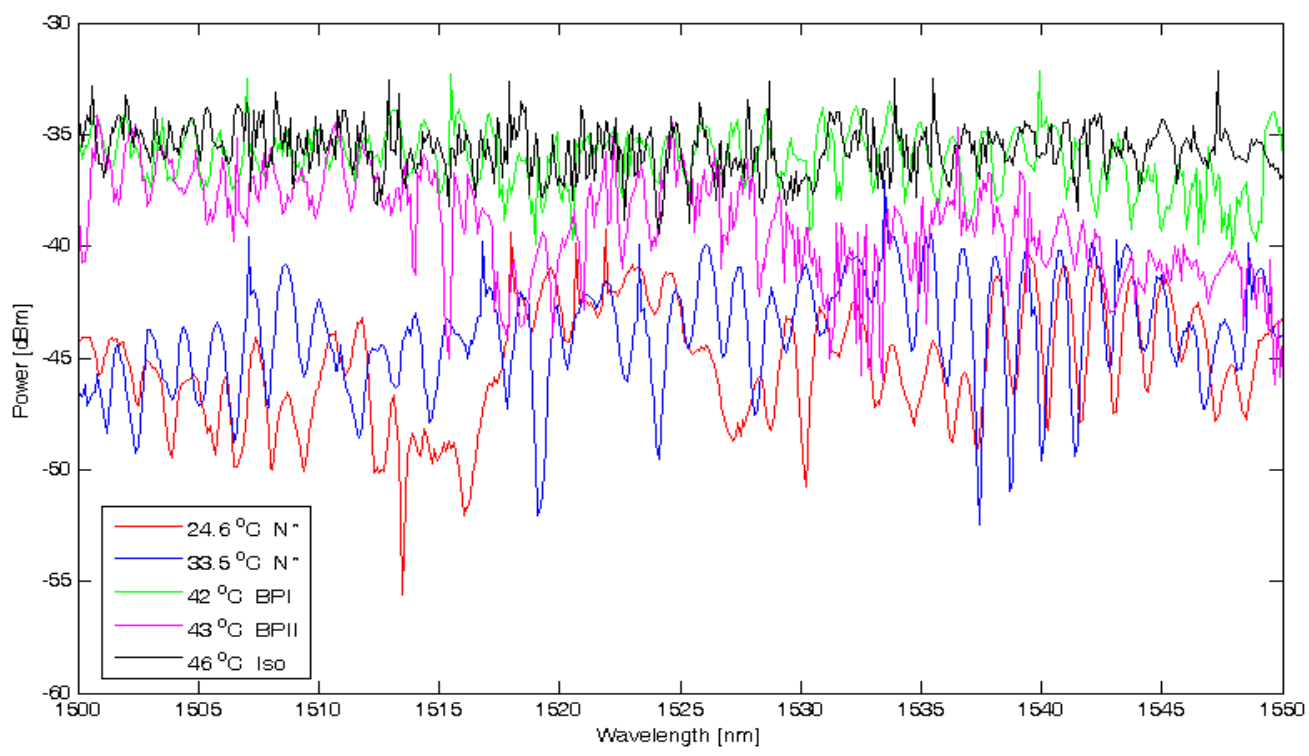

Figure 6: thermal tuning of the guided light of a tunable laser in a PM-1550 selectively filled with cholesteric LC

The pattern is presumably produced by intermodal interferometry, which both period and phase vary with temperature due to changes in the effective refractive index of the liquid crystal. It has been noticed that the light intensity increased when the blue phase apperead, but the signal became noisy and the interference pattern almost disappeared in blue phase/isotropic phase. It is not clear why the interferometry pattern disappear. One reasonable explanation could be that thermal diffusion produces an internal movement of the molecules. 


\section{CONCLUSIONS}

A PCF has been infiltrated selectively with a cholesteric liquid crystal in blue phase. It has been seen that the blue phase has an isotropic behavior in visible region and that the intensity of the guided light increases with temperature in both: visible and near-infrared regions. Further study on the molecules alignment inside the holes of the PCF and on blue

phase stabilization in a broader range of temperature are required. A perspective hybrid PCF-blue phase device could be useful in the development of an in-fiber-polarization controller or a tunable interferometer.

\section{ACKNOWLEDGEMENTS}

This work was supported by the COST Action IC1208 under the STSM reference COST-STSM-ECOST-STSM-IC1208100314-042171. Some of the authors (KO, MT, TRW) gratefully acknowledge support of the NCN grant no. 2011/01/B/ST7/05015.

\section{REFERENCES}

[1] Knight, J. C., Birks, T. A., Russell, P. S. J.., Atkin, D. M., “All-silica single-mode optical fiber with photonic crystal cladding," Opt. Lett. 21(19), 1547-1549 (1996).

[2] Knight, J. C., "Photonic crystal fibres," Nature 424(6950), 847-851 (2003).

[3] Larsen, T., Bjarklev, A., Hermann, D.., Broeng, J., "Optical devices based on liquid crystal photonic bandgap fibres," Opt. Express 11(20), 2589-2596 (2003).

[4] Budaszewski, D., Woliński, T. R., Geday, M.., Otón, J. M., "Photonic Crystal Fibers infiltrated with Ferroelectric Liquid Crystals," Photonics Lett. Pol. 2(3), pp-110 (2010).

[5] Poudereux, D., Corredera, P., Otón, E., Otón, J. M.., Arregui, X. Q., "Photonic liquid crystal fiber intermodal interferometer," Opt. Pura Apl. 46(4), 321-325 (2013).

[6] Woliński, T. R., Szaniawska, K., Ertman, S., Lesiak, P., Domanski, A. W., Dabrowski, R., NowinowskiKruszelnicki, E.., Wojcik, J., "Influence of temperature and electrical fields on propagation properties of photonic liquid-crystal fibres," Meas. Sci. Technol. 17(5), 985 (2006).

[7] Tefelska, M. M., Woliński, T. R., Dąbrowski, R.., Wójcik, J., "Chiral nematic liquid crystals as an alternative filling in photonic crystal fibers," Photonics Lett. Pol. 2(1), pp. 28-30 (2010).

[8] Kutnjak, Z., Garland, C. W., Schatz, C. G., Collings, P. J., Booth, C. J.., Goodby, J. W., "Critical point for the blue-phase-III-isotropic phase transition in chiral liquid crystals," Phys. Rev. E 53(5), 4955 (1996).

[9] Wang, C.-T., Jau, H.-C.., Lin, T.-H., "Bistable cholesteric-blue phase liquid crystal using thermal hysteresis," Opt. Mater. 34(1), 248-250 (2011).

[10] Lee, C.-H., Wu, C.-W., Chen, C.-W., Jau, H.-C.., Lin, T.-H., "Polarization-independent bistable light valve in blue phase liquid crystal filled photonic crystal fiber," Appl. Opt. 52(20), 4849-4853 (2013).

[11] Khoo, I. C., Hong, K. L., Zhao, S., Ma, D.., Lin, T.-H., "Blue-phase liquid crystal cored optical fiber array with photonic bandgaps and nonlinear transmission properties," Opt. Express 21(4), 4319-4327 (2013).

[12] Enz, E., Lagerwall, J., "Electrospun microfibres with temperature sensitive iridescence from encapsulated cholesteric liquid crystal," J. Mater. Chem. 20(33), 6866-6872 (2010).

[13] Mileńko, K., Rutkowska, K. A.., Woliński, T. R., "Numerical and Experimental Analysis of Photonic Crystal Fiber Selectively Infiltrated with Silicon Oil,” Acta Phys. Pol. A 124(3), 589-591 (2013).

[14] Mileńko, K., Woliński, T. R., Shum, P. P., Hu, D. J. J., Lim, J. L.., Yixin, W., "Hybrid photonic crystal fiber selectively infiltrated with liquid crystal,” Photonics Glob. Conf. PGC 2012, 1-3 (2012).

[15] Huang, Y., Xu, Y., Yariv, A., "Fabrication of functional microstructured optical fibers through a selective-filling technique," Appl. Phys. Lett. 85(22), 5182-5184 (2004).

[16] Ertman, S., Czapla, A., Woliński, T. R., Nasiłowski, T., Thienpont, H., Nowinowski-Kruszelnicki, E., Dąbrowski, R., "Light propagation in highly birefringent photonic liquid crystal fibers," Opto-Electron. Rev. 17(2), 150-155 (2009). 